TITLE:

\title{
Single crystal growth of the metallic triangular-lattice antiferromagnet $\mathrm{PdCrO} 2$
}

\author{
$\operatorname{AUTHOR}(\mathrm{S}):$ \\ Takatsu, Hiroshi; Maeno, Yoshiteru
}

\section{CITATION:}

Takatsu, Hiroshi ... [et al]. Single crystal growth of the metallic triangular-lattice antiferromagnet PdCrO2. Journal of Crystal Growth 2010, 312(23): 3461-3465

\section{ISSUE DATE:}

2010-11-15

URL:

http://hdl.handle.net/2433/131816

\section{RIGHT:}

C 2010 Elsevier B.V.; この論文は出版社版でありません。引用の際には 出版社版をご確認ご利用ください。; This is not the published version. Please cite only the published version. 


\title{
Single crystal growth of the metallic triangular-lattice antiferromagnet $\mathrm{PdCrO}_{2}$
}

\author{
Hiroshi Takatsu, Yoshiteru Maeno \\ Department of Physics, Graduate School of Science, Kyoto University, Kyoto 606-8502, Japan
}

\begin{abstract}
We report details of single crystal growth of the metallic triangular-lattice antiferromagnet $\mathrm{PdCrO}_{2}$ consisting of layers of $\mathrm{Pd}$ triangles and $\mathrm{Cr}$ triangles stacking along the $c$ axis. We used the $\mathrm{NaCl}$ flux method and obtained the crystals with the size as large as $2 \times 3.5 \times 0.3 \mathrm{~mm}^{3}$. We confirmed that single crystals have the delafossite structure with the $R \overline{3} m$ symmetry. The electrical resistivity along the $c$ axis and that in the $a b$ plane exhibit metallic temperature dependence with the anisotropic ratio $\rho_{c} / \rho_{a b}$ of over 300 at low temperatures. The residual resistivity of as small as $\rho_{0, a b}=45 \mathrm{n} \Omega \mathrm{cm}$ and the residual resistivity ratio of over 100 indicate high quality of the crystals investigated. Nevertheless, there is no sign of superconductivity down to $0.3 \mathrm{~K}$. These crystals are useful for the investigation of anisotropic magnetic and transport properties including the unconventional anomalous Hall effect (AHE).
\end{abstract}

Key words: A1. Low dimensional structures, A2. Single crystal growth, B1. Oxides, B2. Magnetic materials PACS: 61.66.Fn, 72.15.Eb, 72.80.Ga, 75.30.Gw,

\section{Introduction}

Delafossites $\mathrm{ABO}_{2}$ (A: noble-metal elements; $B$ : transition-metal and rare-earth elements) crystallize in a layered structure consisting of alternating stacks of triangular lattices (TL) of monovalent $A^{1+}$ ions and trivalent $B^{3+}$ ions along the $c$ axis (Fig. 1). Because of the simple TL structure, members with the magnetic ions at the $B$ site are useful for the clarification of the unresolved issues of ideal TL magnets $[1,2,3$, 4]. In addition, by the choice of $A$ site ions, the metallicity can be controlled. Insulating delafossites are actively investigated as the typical multiferroic materials. For examples, $\mathrm{CuFeO}_{2}[5]$ and $\mathrm{CuCrO}_{2}[6,7]$ are known to exhibit strong coupling between the spiral magnetic ordering with the proper-screw type spin structure and the ferroelectric polarization. Metallic delafossites with frustrated spin structure are promising for the investigation of the unconventional anomalous Hall effect (UAHE), which emerges not from the conventional order parameter magnetization $M$ but possibly from the multi-spin quantity "scalar spin chirality" [8].

Although most delafossites are insulators or semiconductors, there are several members of metallic delafossites, such as $\mathrm{AgNiO}_{2}[9,10], \mathrm{PdCoO}_{2}[11,12]$, $\mathrm{PtCoO}_{2}[13,14]$, and $\mathrm{PdCrO}_{2}$ [15]. Among them, only $\mathrm{AgNiO}_{2}$ and $\mathrm{PdCrO}_{2}$ are magnetic metals. In particular, since metallic and magnetic origins are well sepaPreprint submitted to Journal of crystal growth (a)

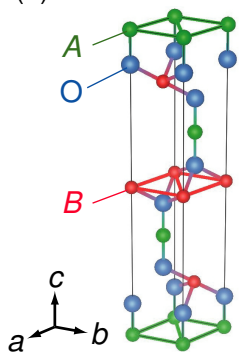

(b)

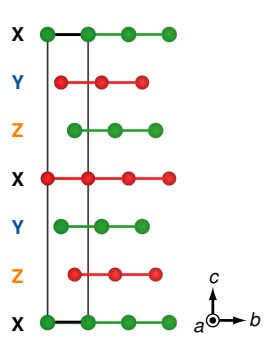

(c)

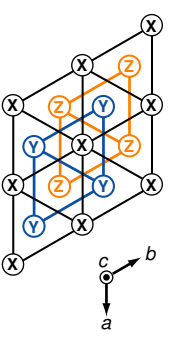

Figure 1: (a) Unit-cell crystal structure of delafossite $A B \mathrm{O}_{2}$. Both $A$ and $B$ ions form triangular lattices and stack along the $c$-axis in the sequence $B-O-A-O-B$. (b) Side view of the crystal structure. The oxygen ions above and below the $A$-site ions are not shown in this figure in order to focus on the layered structure of $A$ and $B$ atoms. The black lines indicate the unit cell. The symbols $\mathrm{X}, \mathrm{Y}$, and $\mathrm{Z}$ represent the staking patterns of the layers. (c) Schematic drawing of the top view of the crystal structure. The definition of the symbols $\mathrm{X}, \mathrm{Y}$, and $\mathrm{Z}$ are the same as that in (b). These figures are generated by VESTA [17].

rated in $\mathrm{PdCrO}_{2}$, this compound should provide unique opportunities to study the interplay between the localized frustrated spins and conduction electrons including UAHE [16].

In order to clarify the mechanism of such unconventional transport properties, determinations of the Fermi surfaces and the detailed magnetic structure are neces-

August 17, 2010 
sary. For these measurements, availability of the highquality single crystals is crucial. Here, we present the details of the growth of high-quality $\mathrm{PdCrO}_{2}$ single crystals. To the best of our knowledge, this is the first report of the details of the single crystal growth of $\mathrm{PdCrO}_{2}$.

Previous studies on powder samples of $\mathrm{PdCrO}_{2}$ have revealed that it exhibits an antiferromagnetic transition at $T_{\mathrm{N}}=37.5 \mathrm{~K}$ forming a $120^{\circ}$ spin structure $[18,15]$. This Neel temperature is an order of magnitude lower than the Weiss temperature $\theta_{\mathrm{W}} \simeq-500 \mathrm{~K}$; the frustration parameter $f \equiv\left|\theta_{\mathrm{W}}\right| / T_{\mathrm{N}}$ is about 13 , indicating a strong frustration among spins. Single crystals obtained in this study revealed isotropic magnetization above $T_{\mathrm{N}}$ consistent with the Heisenberg spins, while they clarified the emergence of anisotropy below $T_{\mathrm{N}}$, indicating that the $120^{\circ}$ spin structure is easy-axis type.

\section{Crystal growth}

Single crystals of $\mathrm{PdCrO}_{2}$ were grown by a $\mathrm{NaCl}$ flux method. Polycrystalline $\mathrm{PdCrO}_{2}$ used for the single crystal growth were prepared in two steps. In the first step, $\mathrm{LiCrO}_{2}$ was prepared as a precursor by the solidstate reaction of the stoichiometric mixture of $\mathrm{Li}_{2} \mathrm{CO}_{3}$ (99.99\%, Aldrich Chemical Co.) and $\mathrm{Cr}_{2} \mathrm{O}_{3}$ (99.99\%, Rare Metallic Co. Ltd.) at $850^{\circ} \mathrm{C}$ in air in an alumina crucible for 24 hours. In the second step, $\mathrm{PdCrO}_{2}$ powder was synthesized by the following metathetical reaction: $\mathrm{Pd}+\mathrm{PdCl}_{2}+2 \mathrm{LiCrO}_{2} \rightarrow 2 \mathrm{PdCrO}_{2}+$ $2 \mathrm{LiCl}[13,15,19]$. For this step, Pd powder $(99.99 \%$, Furuuchi Chem. Co.) and $\mathrm{PdCl}_{2}$ powder $(99.999 \%$, Aldrich Chemical Co.) were used. The mixture was ground in a mortar for 30-60 minutes, sealed in an evacuated quartz tube $(100 \mathrm{~mm} \times \phi 10 \mathrm{~mm})$, heated to $790^{\circ} \mathrm{C}$ and kept at this temperature for 96 hours. The obtained product was washed with aqua regia and distilled water to remove $\mathrm{LiCl}$, unreacted $\mathrm{Pd}$, and other by-products.

Single crystals were grown from the mixture of polycrystals of $\mathrm{PdCrO}_{2}$ and $\mathrm{NaCl}$ (99.99\%, Rare Metallic Co. Ltd.) with a mass ratio of typically $1: 10$. We note here that the minimum mass ratio in which single crystals were formed was $1: 4$. The mixture was sealed in an evacuated quartz tube $(130 \mathrm{~mm} \times \phi 20 \mathrm{~mm})$, heated to $880^{\circ} \mathrm{C}$ in 3.5 hours, and kept at this temperature for 24 hours. It was then cooled down to $800^{\circ} \mathrm{C}$ at the cooling rate of $0.25-0.5^{\circ} \mathrm{C} / \mathrm{h}$ and to $700^{\circ} \mathrm{C}$ at $1^{\circ} \mathrm{C} / \mathrm{h}$. We should note that the melting point of $\mathrm{NaCl}$ is $801^{\circ} \mathrm{C}$ at ambient pressure. After the slow cooling processes, the quartz tube was furnace-cooled down to room temperature. Single crystals were obtained with by-products
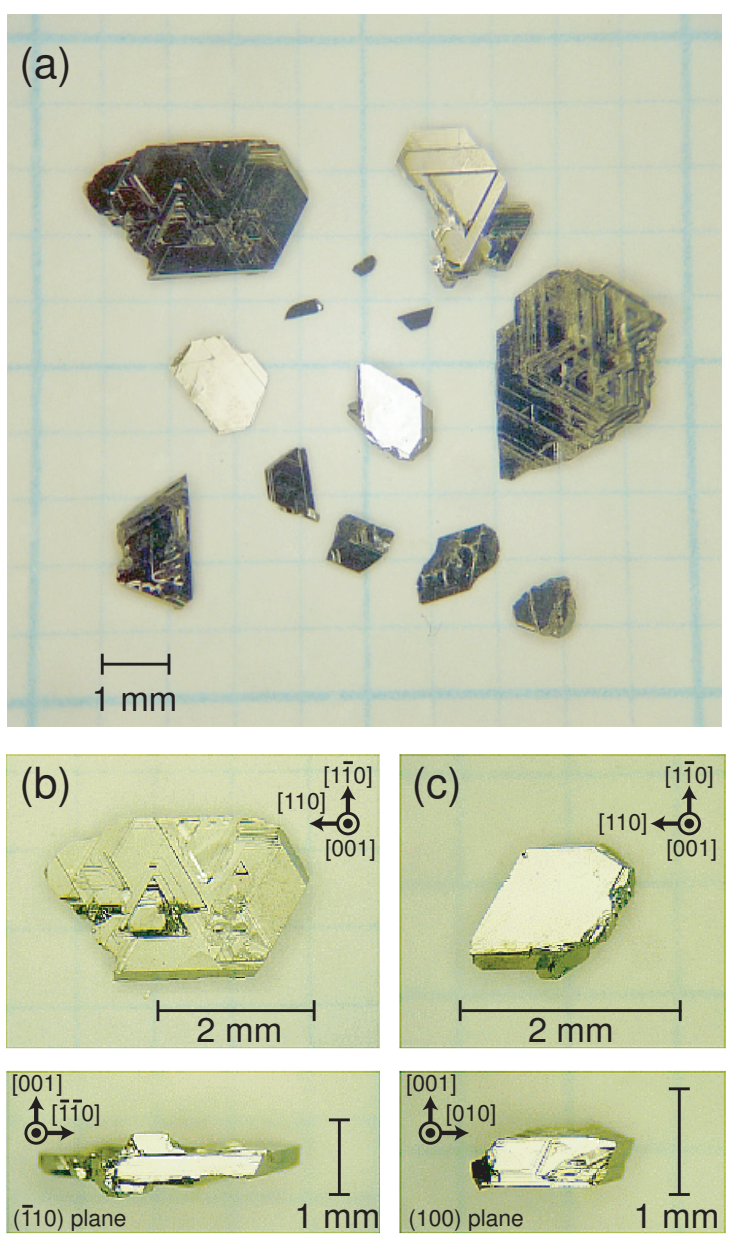

Figure 2: Optical microscope images of as-grown single crystals of $\mathrm{PdCrO}_{2}$.

such as $\mathrm{Cr}_{2} \mathrm{O}_{3}$. In order to grow larger crystals, single crystals were added in the mixture as seeds. The largest crystal size obtained is about $2.0 \times 3.5 \times 0.3 \mathrm{~mm}^{3}$ (see the microscope pictures in Fig. 2). We note here that single crystals of $\mathrm{PdCrO}_{2}$ were not obtained either with $\mathrm{PdCl}_{2}$ flux, $\mathrm{Bi}_{2} \mathrm{O}_{3}$ flux, or $\mathrm{Bi}_{2} \mathrm{O}_{3}-\mathrm{B}_{2} \mathrm{O}_{3}$ mixed flux. The starting $\mathrm{PdCrO}_{2}$ polycrystals remained unreacted in $\mathrm{PdCl}_{2}$ flux. They decomposed in $\mathrm{Bi}_{2} \mathrm{O}_{3}$ and $\mathrm{Bi}_{2} \mathrm{O}_{3}-$ $\mathrm{B}_{2} \mathrm{O}_{3}$ fluxes but did not produce any crystals. We also note that attempts to grow the single crystals in air using Pt crucibles were not successful.

\section{Characterization}

Figure 3 represents the powder X-ray diffraction (XRD) spectra of polycrystalline and single-crystalline samples of $\mathrm{PdCrO}_{2}$, with the $\mathrm{CuK}_{\alpha 1}$ radiation. These 


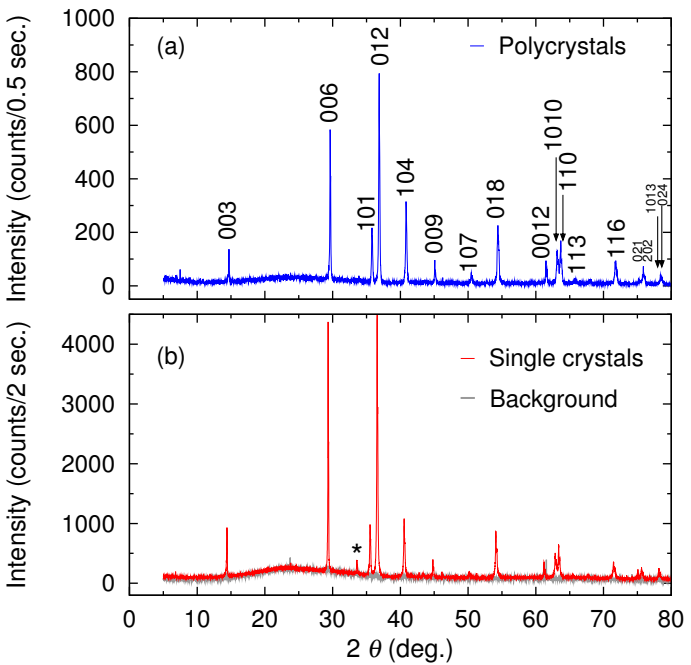

Figure 3: Powder XRD spectra of (a) polycrystalline samples and (b) crushed single-crystalline samples of $\mathrm{PdCrO}_{2}$ at room temperature. The peak pattern can be labeled with the delafossite structure with $R \overline{3} m$ symmetry. The additional peak labeled with $*$ for the single crystal spectrum at about $2 \theta=34^{\circ}$ originates from an impurity phase of $\mathrm{Cr}_{2} \mathrm{O}_{3}$. It is well separated from the $\mathrm{PdCrO}_{2}$ single crystals, but a small amount of powder $\mathrm{Cr}_{2} \mathrm{O}_{3}$ was inevitably picked up in the preparation for measurements.

two spectra exhibit the same patterns, yielding the lattice parameters of $a=b=2.923 \AA, c=18.086 \AA$ for both single crystals and polycrystals.

The X-ray Laue patterns for the (001) plane and (100) plane are presented in Figs. 4(a) and (b). Calculated patterns for these conditions shown in Figs. 4(c) and (d) are consistent with the measured patterns. The clear Laue spots guarantee high crystallinity of the crystals. Scanning electron microscope (SEM) images with the energy dispersive X-ray (EDX) analysis mapping are presented in Fig. 5. The EDX analyses confirmed the homogeneity of the crystals with the composition ratio of $\mathrm{Pd} / \mathrm{Cr}=1.1 \pm 0.1$. Fig. 5 also indicates the $\mathrm{Cr}_{2} \mathrm{O}_{3}$ impurity phase localized on the left edge of the crystal.

Figure 6(a) represents the electrical resistivity along the $c$ axis $\left(\rho_{c}\right)$ and in the $a b$ plane $\left(\rho_{a b}\right)$ measured with both the ac and dc four-probe methods from $300 \mathrm{~K}$ to $0.32 \mathrm{~K}$. Both $\rho_{c}$ and $\rho_{a b}$ exhibit metallic temperature dependence down to the lowest measurement temperatures with clear anomalies at $T_{\mathrm{N}}$. The residual resistivities are $\rho_{0, c}=16.5 \mu \Omega \mathrm{cm} \rho_{0, a b}=45 \mathrm{n} \Omega \mathrm{cm}$ (see the inset of Fig. 6). The residual resistivity ratio, $\mathrm{RRR} \equiv \rho(295 \mathrm{~K}) / \rho(0.32 \mathrm{~K})$, is estimated to be 104 for $\rho_{c}$ and 200 for $\rho_{a b}$. By using the value of the Fermi wave number $k_{\mathrm{F}}=0.93 \AA^{-1}$ [20] and the carrier den-
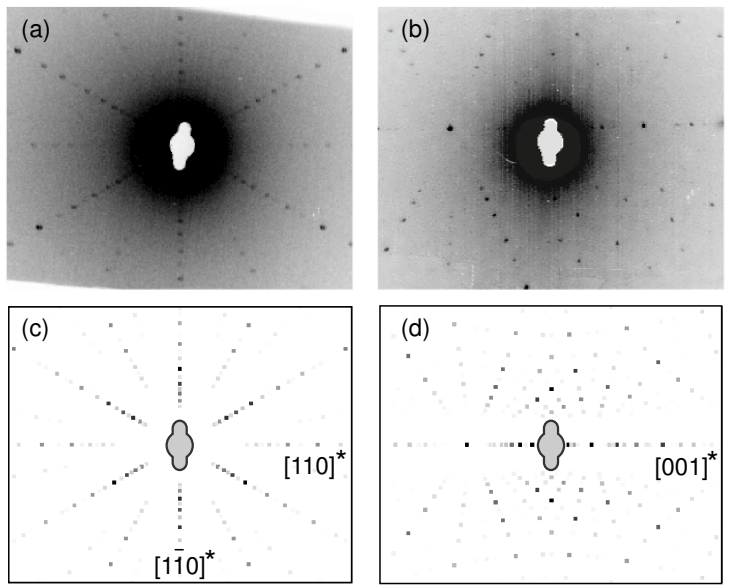

Figure 4: X-ray Laue patterns of (a) the (001) plane and (b) the (100) plane in back-scattered conditions. The photos exhibit clear spots that agree with the $R \overline{3} m$ symmetry. (c) and (d): the corresponding calculated patterns.

sity $n=1.62 \times 10^{22} \mathrm{~cm}^{-3}$ [21], the mean free path $l=\left(\hbar k_{\mathrm{F}} / n e^{2} \rho_{0}\right)$ in the $a b$ plane is estimated to be as large as $l_{a b} \simeq 30 \mu \mathrm{m}$. These large RRR and $l$ guarantee high quality of the obtained crystals. The anisotropic ratio $\rho_{c} / \rho_{a b}$ is over 190 in the whole measurement temperature range. The large anisotropy of the resistivity is consistent with the expectation that the metallic conductivity mainly originates from the Pd layer.

The dc magnetization $M$ was measured with a SQUID magnetometer (Quantum Design, MPMS) from $300 \mathrm{~K}$ to $1.8 \mathrm{~K}$ in magnetic fields $\mu_{0} H$ between 0.01 and $7 \mathrm{~T}$ in both field-cooled (FC) and zero field cooled (ZFC) conditions. Figure 6(b) shows the temperature dependence of the magnetic susceptibilities $(\chi=M / H)$ measured in the field $\mu_{0} H=1 \mathrm{~T}$ along the $c$ axis, and in the $a b$ plane. Above $T_{\mathrm{N}}, \chi$ is isotropic, exhibiting the Curie-Weiss behavior above $200 \mathrm{~K}$ with the localized moment $\mu_{\mathrm{eff}}=4.0 \pm 0.2 \mu_{\mathrm{B}}$ [15]. These results suggest that $\mathrm{PdCrO}_{2}$ constitutes a Heisenberg spin system, consistent with the expectation of the localized spins of the $\mathrm{Cr}^{3+}$ ion $\left(3 \mathrm{~d}^{3}, S=3 / 2\right)$. In contrast, below $T_{\mathrm{N}}$, at which the spins form a $120^{\circ}$ structure $[15,18]$, $\chi$ becomes anisotropic with a sudden decrease at $T_{\mathrm{N}}$; the value of $\chi_{a b}$ becomes larger than that of $\chi_{c}$. Such anisotropy is similar to the susceptibility of another delafossite $\mathrm{CuCrO}_{2}$ [7], but is different from a similar layered TL magnet $\mathrm{LiCrO}_{2}$ with $\chi_{c}$ larger than $\chi_{a b}$ [22]. Within the mean field approximation [23], $\chi$ should be isotropic even below $T_{\mathrm{N}}$ if the inter-plane spin configuration is ferromagnetic. The observed anisotropy 

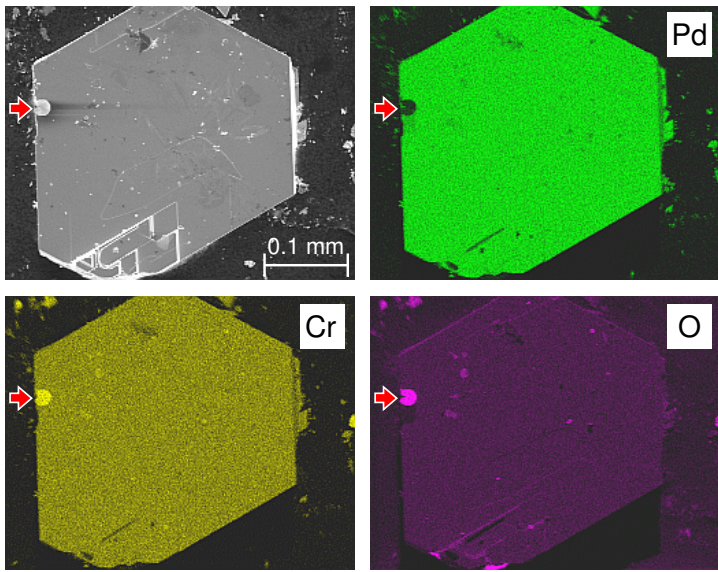

Figure 5: EDX mapping in SEM images of single crystalline $\mathrm{PdCrO}_{2}$. The coloring of the images represents the concentration of elements constituting $\mathrm{PdCrO}_{2}$ : $\mathrm{Pd}$ (Green), $\mathrm{Cr}$ (Yellow) and $\mathrm{O}$ (Purple). The $\mathrm{Cr}_{2} \mathrm{O}_{3}$ impurity can be seen as a separated phase at the left edge of the crystal (Red arrows).

antiferromagnetic and the spins lie in a plane containing the $c$ axis (the so-called easy-axis type $120^{\circ}$ structure). We note that the anisotropy $\chi_{a b}<\chi_{c}$ for $\mathrm{LiCrO}_{2}$ is attributed to a $120^{\circ}$ structure where the spins lie in the $a b$ plane (the easy-plane type structure) with an antiferromagnetic inter-plane configuration.

\section{Conclusion}

We have succeeded in growing single crystals of $\mathrm{PdCrO}_{2}$ by a $\mathrm{NaCl}$ flux method. It was confirmed that the obtained crystals are in the delafossite structure with the $R \overline{3} m$ symmetry. The large values of the residual resistivity ratio over 100 and the mean free path in the $a b$ plane as large as $30 \mu \mathrm{m}$ guarantee the high quality of the crystals.

The metallic resistivity with a large anisotropy $\left(\rho_{c} / \rho_{a b}>190\right)$ supports the expectation that the conductivity in $\mathrm{PdCrO}_{2}$ mainly originates from the $\mathrm{Pd}$ layers. The magnetic susceptibility above $T_{\mathrm{N}}$ confirms that $\mathrm{PdCrO}_{2}$ constitutes a Heisenberg spin system. Moreover, the emergence of the anisotropy at $T_{\mathrm{N}}$ indicates an antiferromagnetic coupling between the layers with the easy-axis type $120^{\circ}$ spin structure.

These crystals make possible further investigations of the details of both the actual spin configuration and the Fermi surface topology, as well as searches of other fascinating phenomena related to the frustrated magnetism.
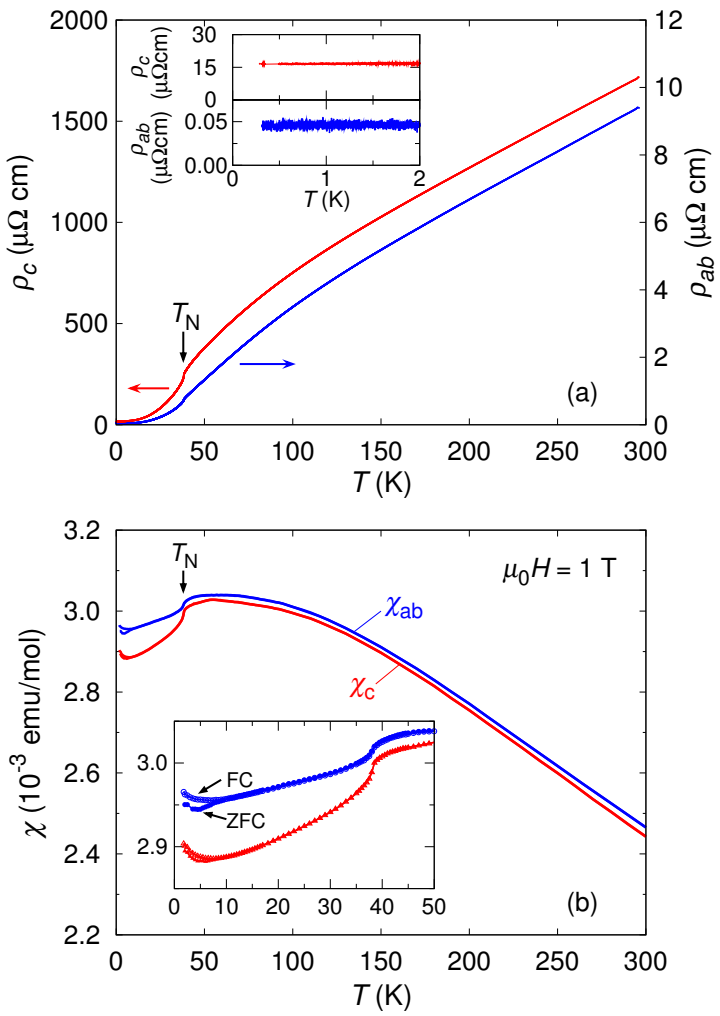

Figure 6: (a) Temperature dependence of the electrical resistivity along the $c$ axis $\left(\rho_{c}\right)$ and in the $a b$ plane $\left(\rho_{a b}\right)$. Both $\rho_{c}$ and $\rho_{a b}$ exhibit clear anomalies at $T_{\mathrm{N}}$, associated with the antiferromagnetic transition. The inset shows the low temperature behavior of $\rho_{c}$ and $\rho_{a b}$. (b) Temperature dependence of the magnetic susceptibilities in the field at $1 \mathrm{~T}$ along the $c$ axis $\left(\chi_{c}\right)$ and in the $a b$ plane $\left(\chi_{a b}\right)$. Above $T_{\mathrm{N}}, \chi_{c}$ and $\chi_{a b}$ are nearly equal. In contrast, below $T_{\mathrm{N}}, \chi_{c}$ becomes smaller than $\chi_{a b}$. The inset focuses on the behavior near and below $T_{\mathrm{N}}$. A slight splitting of the ZFC and FC curves appears at temperatures below about $10 \mathrm{~K}$.

\section{Acknowledgements}

We would like to thank J. Ishikawa for his assistance of the single crystal growth. We also thank K. Ishida, S. Yonezawa, M. Kriener, D. Peets, Y. Nakai and S. Kittaka for useful discussions and for their experimental assistance. This work was supported by the Grant-in-Aid for the Global COE Program "The Next Generation of Physics, Spun from Universality and Emergence" from the Ministry of Education, Culture, Sports, Science and Technology (MEXT) of Japan and Grants-in-Aids for Scientific Research from the Japan Society for the Promotion of Science (JSPS). H.T. is financially supported as a JSPS research fellow. 


\section{References}

[1] G. H. Wannier, Phys. Rev. 79 (1950) 357.

[2] P. W. Anderson, Mater. Res. Bull. 8 (1973) 153

[3] S. Miyashita, H. Shiba, J. Phys. Soc. Jpn. 53 (1984) 1145.

[4] H. Kawamura, S. Miyashita, J. Phys. Soc. Jpn. 53 (1984) 4138.

[5] T. Kimura, J. C. Lashley, A. P. Ramirez, Phys. Rev. B 73 (2006) 220301(R).

[6] S. Seki, Y. Onose, Y. Tokura, Phys. Rev. Lett. 101 (2008) 067204.

[7] K. Kimura, H. Nakamura, K. Ohgushi, T. Kimura, Phys. Rev. B 78 (2008) 140401(R).

[8] N. Nagaosa, J. Phys. Soc. Jpn. 75 (2006) 042001.

[9] A. Wichainchi, P. Dordor, J. P. Doumerc, E. Marquestaut, M. Pouchard, P. Hagenmuller, J. Solid State Chem. 74 (1988) 126.

[10] E. Wawrzyńska, R. Coldea, E. M. Wheeler, I. I. Mazin, M. D. Johannes, T. Sörgel, M. Jansen, R. M. Ibberson, P. G. Radaelli, Phys. Rev. Lett. 99 (2007) 157204.

[11] H. Takatsu, S. Yonezawa, S. Mouri, S. Nakatsuji, K. Tanaka, Y. Maeno, J. Phys. Soc. Jpn. 76 (2007) 104701.

[12] H.-J. Noh, J. Jeong, J. J. E.-J. Cho, S. B. Kim, K. Kim, B. I. Min, H. D. Kim, Phys. Rev. Lett. 102 (2009) 256404.

[13] R. D. Shannon, D. B. Rogers, C. T. Prewitt, Inorg. Chem. 10 (1971) 713 .

[14] M. Itoh, M. Mori, M. Tanaka, H. Takei, Physica B 259-261 (1999) 999.

[15] H. Takatsu, H. Yoshizawa, S. Yonezawa, Y. Maeno, Phys. Rev. B 79 (2009) 104424.

[16] H. Takatsu, S. Yonezawa, S. Fujimoto, Y. Maeno (in press).

[17] K. Momma, F. Izumi, J. Appl. Crystallogr. 41 (2008) 653.

[18] M. Mekata, T. Sugino, A. Oohara, Y. Oohara, H. Yoshizawa, Physica B 213 (1995) 221.

[19] J. P. Doumerc, A. Wichainchai, A. Ammar, M. Pouchard, P. Hagenmuller, Mat. Res. Bull. 21 (1986) 745.

[20] T. Shishidou and T. Oguchi, (Personal communication). Here, the value of $k_{\mathrm{F}}$ is estimated from the band structure calculation.

[21] The carrier density $n$ is estimated from the ordinary Hall coefficient $R_{0}$ with the single band approximation, $R_{0}=1 / n e$. It is however difficult to evaluate $R_{0}$ of $\mathrm{PdCrO}_{2}$ because the Hall resistivity in $\mathrm{PdCrO}_{2}$ contains the anomalous Hall resistivity in addition to the ordinary Hall resistivity. Thus, $R_{0}$ of $\mathrm{PdCrO}_{2}$ is approximated by $R_{0}$ of the isostructural nonmagnetic compound $\mathrm{PdCoO}_{2}$ [16]. This approximation is based on the expectation that one $\mathrm{Pd} 4 \mathrm{~d}$ electron per formula unit dominantly contributes to the electric conductivity for both $\mathrm{PdCrO}_{2}$ and $\mathrm{PdCoO}_{2}$. Indeed, the electronic specific heat coefficient, related to the density of states of conduction carriers, is nearly the same between these two oxides.

[22] H. Kadowaki, H. Takei, K. Motoya, J. Phys. Cond. Matt. 7 (1995) 6869.

[23] T. Hirone, K. Adachi, J. Phys. Soc. Jpn. 12 (1957) 156. 